\title{
Sciendo
}

DOI: 10.1515/aon-2019-0011

\section{RELIABILITY TEST OF A GNSS ONBOARD RECEIVER IN THE KINEMATIC MODE}

\author{
Janusz Ćwiklak \\ Polish Air Force Univerisity, Faculty of Aviation \\ Dywizjonu Street 303 nr 35, 08-521 Dęblin, Poland \\ e-mail:j.cwiklak@wsosp.pl \\ Kamil Krasuski \\ Polish Air Force University, Faculty of Aviation, Poland \\ Dywizjonu Street 303 nr 35, 08-521 Dęblin, Poland \\ e-mail: kk_deblin@wp.pl \\ Marek Grzegorzewski \\ Polish Air Force Univerisity, Faculty of Aviation \\ Dywizjonu Street 303 nr 35, 08-521 Dęblin, Poland \\ e-mail:m.grzegorzewski@wsosp.pl
}

\begin{abstract}
The article presents the results of the aircraft Cessna 172 positioning based on navigation solutions in the GPS and EGNOS (SBAS) tracking mode. The article makes a comparison of coordinate readings of the Cessna 172 in the ellipsoidal BLh frame. The verification of the coordinates of the aircraft Cessna 172 was used to assess the reliability of the GNSS satellite technique in aviation. In a research test, the navigation data were recorded by the onboard receiver Thales Mobile Mapper during an air test performed over the military aerodrome EPDE in Dęblin. Judging by the conducted investigations, it is possible to conclude that the difference in BLh coordinates of the aircraft Cessna 172 on the basis of the GPS solution and EGNOS (SBAS) solution equals, respectively: from $-0.5 \mathrm{~m}$ to $+3 \mathrm{~m}$ for component $\mathrm{B}$; and from $-2 \mathrm{~m}$ to $+6 \mathrm{~m}$ for component $\mathrm{L}$; from approximately $-11 \mathrm{~m}$ to over $+1 \mathrm{~m}$ for component $\mathrm{h}$. In addition, the paper defines factors of dilution of precision PDOP, based on the GPS and EGNOS (SBAS) solutions. The average value of the PDOP coefficient for a solution in the tracking GPS mode was 2.7, whereas in the EGNOS (SBAS) tracking mode, it was equal to 2.8 .
\end{abstract}

\section{Keywords - GPS, EGNOS, RMS, air navigation, linear regression}

\section{INTRODUCTION}

GNSS satellite technology guarantees a high positioning accuracy of aircraft in the air navigation area. It needs to be stressed that the parameter of accuracy is one of the elements determining the quality of satellite positioning in air navigation. Other parameters are availability, continuity and reliability of satellite positioning of air navigation services [1]. It is worth noting that in terms of the use of GNSS satellite technology in aviation, there also appears the concept of reliability. Reliability, in the area of air navigation, is defined as a number of independent supernumerary measurements for the correct designation of navigation parameters of an aircraft [2]. Navigation parameters determined from the GNSS satellite technology should be understood as the coordinates of an aircraft, flight speed and angles of orientation Heading, Pitch, Roll in 3D space. The parameter 
of reliability is therefore essential from the standpoint of verification of actual readings of navigation parameters during the execution of flight operations.

While determining the aircraft coordinates, the parameter of aircraft reliability is designed in the aspect of applying multiple GNSS measurement techniques in precision positioning of an aircraft in air navigation. Such an approach allows an optimal adjustment of selected research methods to reproduce a position of an aircraft. In addition, in such a case there is a guarantee of reliability assessment of individual GNSS measurement techniques and an analysis of their usefulness in precise positioning of an aircraft in air navigation. When assessing reliability, the value of a measured parameter in a specified time unit, along with an indication of operating conditions, are given. Within the framework of an aircraft position, the dimension of coordinates referenced to the XYZ geocentric system, ellipsoidal BLh system or the local ENU system [3] are given. The time unit is a system of time reference, usually in GNSS satellite measurements. It is the time of the navigation system GPS- GPS Time (GPST). The operating conditions are expressed by means of a set of internal and external factors, stimulating the measurement process performed by the GNSS satellite receiver.

The control process is an essential element of a correct determination of the reliability parameter. During aircraft operations, GNSS satellite technology offers ample opportunities to verify a position of an aircraft. In air navigation, there are absolute and differential methods of aircraft positioning in real time and in postprocessing. In the case of absolute positioning methods, there are the SPP (single point positioning) and the PPP methods (Precise Point Positioning) used. Among the differential techniques, there are methods of positioning using both a single or double difference in GNSS satellite measurements. The technique of a single difference is applied in the BSSD (Between Satellite Single Difference) and in the method BRSD (Between Receiver Single Difference). The technique of a single difference is applied in the RTK-OTF for phase GNSS measurements and DGNSS method for code measurements [4]. In the process of the examination of the results obtained from a given test method, it is very important to determine the reference system and the reference time for the executed GNSS measurements. Such a solution enables a precise selection of another measurement technique, which will be used to verify the obtained test results. Such an approach ensures determining the convergence of results of the values of a given parameter using two different measurement techniques, which is the essence of reliability in satellite GNSS measurements. Furthermore, the convergence of the value of a given parameter taken in two different measurement techniques should be defined by a numerical interval of a dataset and the basic statistical characteristics. Among the statistical parameters, it is necessary to determine the mean value of a parameter, the median, standard deviation, the minimum and maximum value.

The aim of the presented work is to determine the reliability of the designation of coordinates of the Cessna 172 aircraft in the kinematic mode. The coordinates of the Cessna 172 aircraft were determined in the ellipsoidal BLh system on the basis of the GPS and EGNOS solutions. The navigation data of the Cessna 172 position were registered by the onboard receiver Thales Mobile Mapper mounted in the pilot's cockpit. In the reliability analysis, the authors made a comparison of BLH ellipsoidal coordinate readings of the Cessna 172 for the solutions GPS and EGNOS. The article is divided into 4 parts: introduction, research material, research methods, results and discussion, as well as conclusions. The paper is completed with a list of research literature references.

\section{MATERIALS AND METHODS}

In the framework of the conducted reliability test of determining the position of the airplane Cessna 172, the authors established the aircraft coordinates and made their comparison in the ellipsoidal BLh system. Ultimately, the position of the Cessna 172 was designated with the GPS code observations (method I) as well as using the support system EGNOS (method II). The receiver Thales Mobile Mapper was located in a cockpit during a research experiment for the military airport EPDE in Dęblin. The purpose of the air test was to examine and verify the operation of various onboard GNSS satellite receivers for precise positioning of the aircraft in the real time mode and in post-processing [5]. The receiver Thales Mobile Mapper worked in the kinematic mode during the executed air test, determining the aircraft position in the GPS and EGNOS (SBAS) tracking 
mode. It should be added that the receiver Thales Mobile Mapper can also operate in the tracking mode DGPS with differential corrections. In the case of GPS tracking mode, the receiver Thales Mobile Mapper uses a navigational message and GPS code observations on L1 frequency in determining the position of the Cessna 172 aircraft. In case of the EGNOS tracking mode, the receiver Thales Mobile Mapper uses differential corrections EGNOS (SBAS) in order to determine the position of a Cessna 172 aircraft. It must be noted that the basic technical characteristics of the receiver Thales Mobile Mapper in the tracking GPS and EGNOS mode in order to determine the navigational position can be characterised as follows: operational memory - commonly up to 4 MB RAM (optionally even $32 \mathrm{MB}$ and $64 \mathrm{MB}$ ); receiver internal software: Mobile Mapper Field and Mobile Mapper Office; data export format: SHP, MFIS and DXF; possibility of using GIS tools: yes; possibility of using background maps: yes; reference system: global, standard WGS-84; calculation modes: GPS, EGNOS, DGPS; the maximum number of GPS satellites being tracked: up to 12 GPS satellites; tracking manner of GPS satellites: sequential; initialization of calculations: "cold start" < 2 minutes, "warm start" < 1 minute, "hot start" $<15$ seconds; interval of computations, and time of recording the observations: 1 or 2 seconds; positioning accuracy in EGNOS mode (SBAS): up to $3 \mathrm{~m}$ at 0.95 confidence level; receiver antenna: in-built in the receiver; receiver weight: less than $0.5 \mathrm{~kg}$; battery life: usually up to 8 hours; number of batteries in the set: usually 2 batteries built into the receiver; atmospheric conditions of using the receiver: air temperature from $-10{ }^{\circ} \mathrm{C}$ to 60 ${ }^{\circ} \mathrm{C}[6]$.

\section{RESULTS AND DISCUSSION}

In the first stage of determining the reliability of designing the position of the aircraft the Cessna 172 , the authors determined the values of dilution of precision factors PDOP [7] using the GPS solution and EGNOS solution (SBAS). Figure 1 shows the designated values of the PDOP factors for an individual navigation solution of a position in the GPS and EGNOS (SBAS) tracking modes.

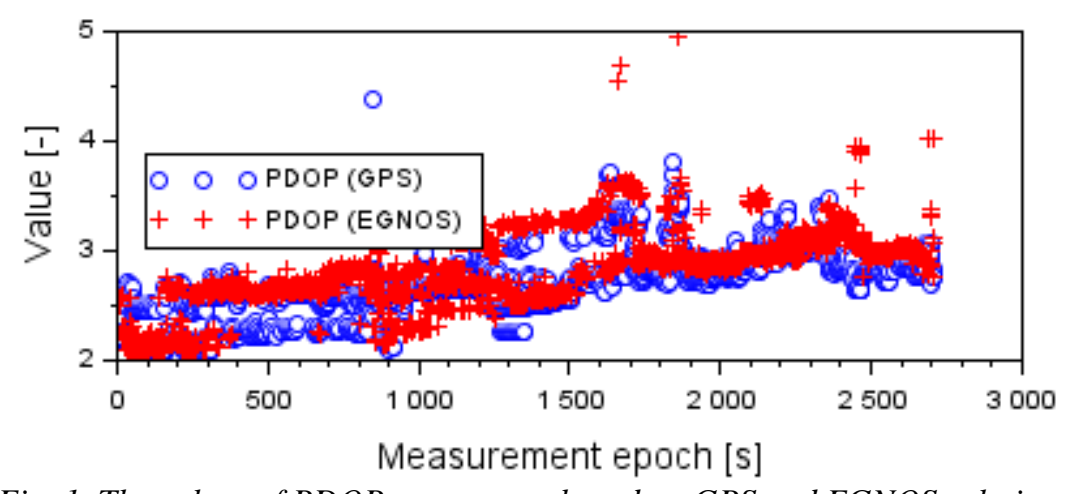

Fig. 1. The values of PDOP parameters based on GPS and EGNOS solution.

The average value of the coefficient PDOP for a solution in the GPS tracking mode was equal to 2.7, with the median also equal to 2.7. Moreover, the dispersion of the obtained results PDOP in the GPS tracking mode ranged from 2.1 to 4.4. The average value of the coefficient PDOP for a solution in the EGNOS (SBAS) tracking mode equalled 2.8, similarly to the statistical parameter of the median. On the other hand, the dispersion of the results obtained in the EGNOS (SBAS) tracking mode ranged from 2.1 to 4.9. It should be emphasized that lower values of the coefficients PDOP were obtained in the GPS solution than in the solution EGNOS (SBAS). In the GPS solution, PDOP factors were lower by 5\% than in the tracking EGNOS (SBAS) mode . It is worth adding that in the GPS tracking, approximately $20 \%$ of all values of coefficients PDOP were below 2.5. Moreover, in the GPS tracking, approximately $87 \%$ of all values of coefficients PDOP were below 3 . On the other hand, in the GPS tracking mode, more than $99 \%$ of all the values of the coefficients PDOP were below 3.5. In the tracking mode, the EGNOS (SBAS), approximately $13 \%$ of all the results of the coefficients PDOP was less than 2.5. Besides, in the tracking mode EGNOS (SBAS), approximately $76 \%$ of all the results of the 
coefficients PDOP was less than the value of 3. In the tracking EGNOS (SBAS) mode, over 97\% of all the results of the coefficients PDOP was less than 3.5.

The second stage of the investigation concerning the determination of the reliability of a position of an aircraft Cessna 172 was to compare the readings of the coordinate value B (Latitude) with GPS and EGNOS (SBAS) solutions . For this purpose, the authors determined a difference in the B element in the GPS and EGNOS (SBAS) solutions, as follows [8]:

$$
\mathrm{dB}=\mathrm{B}_{\mathrm{GPS}}-\mathrm{B}_{\mathrm{EGNOS}}
$$

where:

$\mathrm{dB}$ - difference of B element value in GPS and EGNOS solutions, $\mathrm{B}_{\mathrm{GPS}}$ - B coordinate value in GPS solution;

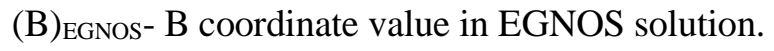

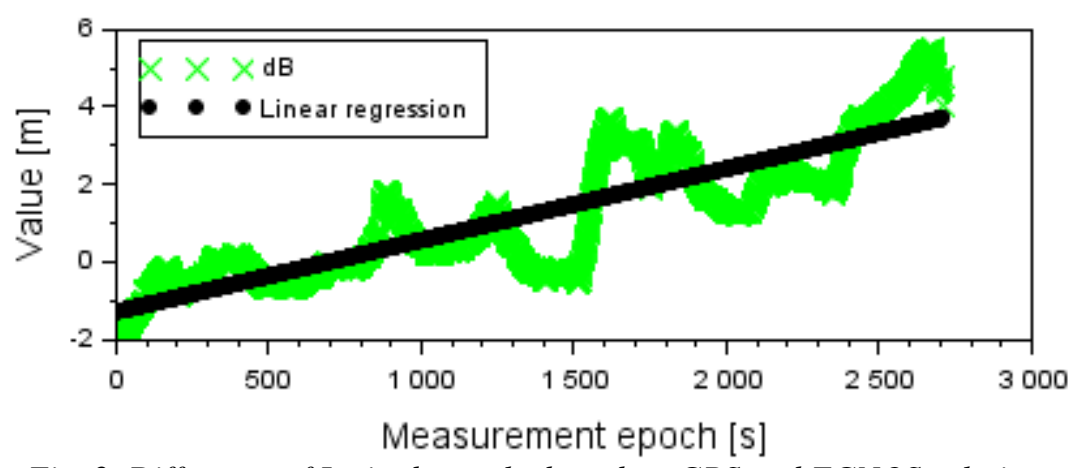

Fig. 2. Difference of Latitude results based on GPS and EGNOS solution.

Figure 2 shows the results of comparing the readings for $\mathrm{B}$ element on the basis of a navigational solution of a position in the tracking GPS and EGNOS (SBAS) modes. The average value of the B element difference on the basis of a comparison of GPS and EGNOS solutions equals $1.17 \mathrm{~m}$, with the statistical median parameter equal to $0.70 \mathrm{~m}$. Furthermore, the dispersion of the obtained results for the parameter $\mathrm{dB}$ ranges from $-1.88 \mathrm{~m}$ to $+5.46 \mathrm{~m}$. It should be added that the precision error RMS [9] for parameter $\mathrm{dB}$ is equal to $1.67 \mathrm{~m}$. It is worth noting that approximately $52 \%$ of all the $\mathrm{dB}$ parameter values is below $1 \mathrm{~m}$. In addition, approximately $83 \%$ of all the $\mathrm{dB}$ parameter values is below $3 \mathrm{~m}$. In turn, over $97 \%$ of all the $\mathrm{dB}$ parameter values is less than $5 \mathrm{~m}$. Figure 2 also specified the trend in changes of the parameter $\mathrm{dB}$ based on the GPS solution and EGNOS solution (SBAS). For this purpose, the function of linear regression for the characteristic of changes of a first degree polynomial was used. The values of linear coefficients $(a, b)$ of the function of linear regression were designated using the least squares method without the introduction of weighted measurements [10]. Therefore, the linear coefficients $(a, b)$ that describe the nature of the changes of parameter dB have the following values: $a=0.001$ $\mathrm{m} / \mathrm{s}$, and (b) =-1.332 m, respectively. In addition, an adjustment error of the function of linear regression against the matching results of $\mathrm{dB}$ parameter is $\mathrm{m}=0.84 \mathrm{~m}$. The distribution of corrections in the least squares method was $-2.01 \mathrm{~m}$ to $+2.05 \mathrm{~m}$.

The third stage of the investigation concerning the determination of reliability of the aircraft Cessna 172 position was to compare the readings of the coordinate value L (Longitude) with GPS and EGNOS (SBAS) solutions. For this purpose, the authors determined the difference in the L element in the GPS and EGNOS (SBAS) solutions, as below [8]:

$$
\mathrm{dL}=\mathrm{L}_{\mathrm{GPS}}-\mathrm{L}_{\mathrm{EGNOS}}
$$

where:

$\mathrm{dL}$ - difference of $\mathrm{L}$ element value in GPS and EGNOS solutions, $\mathrm{L}_{\mathrm{GPS}^{-}}$coordinate value in GPS solution; LEGNOS- L coordinate value in EGNOS solution. 
Figure 3 shows the results of comparing the readings for the L element on the basis of a navigational solution of a position in the tracking GPS and EGNOS (SBAS) mode. The average value of the L element difference on the basis of a comparison of solutions GPS and EGNOS is $0.75 \mathrm{~m}$, with the statistical median parameter equal to $0.58 \mathrm{~m}$. Furthermore, the dispersion of the obtained results for the parameter $\mathrm{dL}$ ranges from $-0.44 \mathrm{~m}$ to $+2.70 \mathrm{~m}$. It should be added that the precision error RMS for parameter $\mathrm{dL}$ is equal to $0.75 \mathrm{~m}$. It is worth noting that approximately $72 \%$ of all the parameter values DL is less than $-1 \mathrm{~m}$. It should be added that approximately $84 \%$ of all the $\mathrm{dL}$ parameter values $\mathrm{dL}$ is less than $1.5 \mathrm{~m}$. In addition, over $93 \%$ of all the parameter values $\mathrm{dL}$ is less than $2 \mathrm{~m}$. Finally, over $99 \%$ of all the parameter values $\mathrm{dL}$ is below $2.5 \mathrm{~m}$. Figure 3 also specifies a trend of dL parameter changes based on the GPS solution and EGNOS solution (SBAS). For this purpose, the function of linear regression for the characteristics of changes of a first degree polynomial was used. The values of linear coefficients $(a, b)$ of the function of linear regression were designated using the least squares method without the introduction of weighted measurements [10]. Therefore, the linear coefficients (a, b) describing the nature of the changes of dL parameter had the following values: $a=0.001 \mathrm{~m} / \mathrm{s}$, and $(\mathrm{b})=0.278$ $\mathrm{m}$, respectively. In addition, an error matching the function of linear regression with regard to the matching results of the parameter $\mathrm{dL}$ is $\mathrm{m}=0.70 \mathrm{~m}$. The distribution of corrections in the least squares method equalled $-1.47 \mathrm{~m}$ up to $+1.54 \mathrm{~m}$.

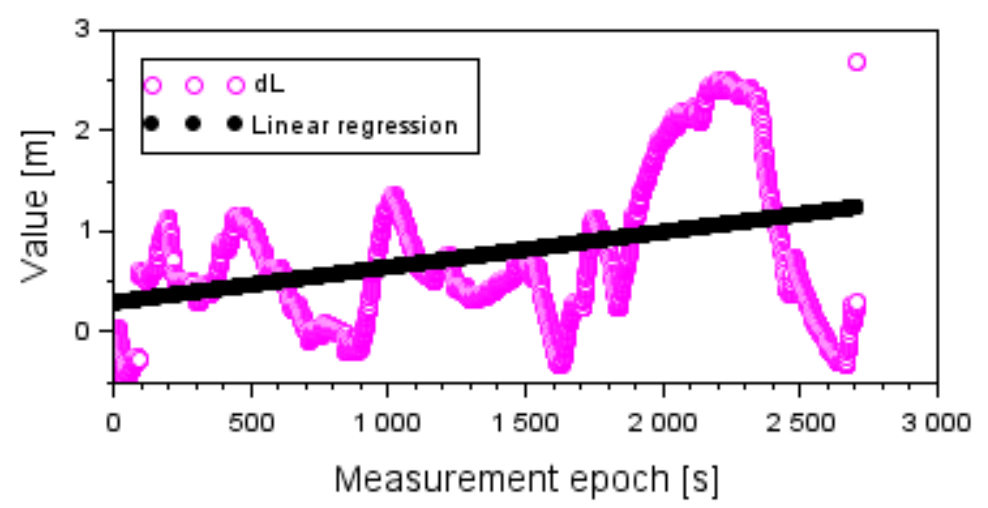

Fig. 3. Difference of Longitude results based on GPS and EGNOS solutions.

The fourth phase of the investigation concerning the determination of the reliability of the aircraft Cessna 172 position was to compare the readings of the coordinate value $h$ (ellipsoidal height) in GPS and EGNOS (SBAS) solutions. For this purpose, the authors determined the difference in the $h$ element in the GPS and EGNOS (SBAS) solutions, as follows [8]:

$$
\mathrm{dh}=\mathrm{h}_{\mathrm{GPS}}-\mathrm{h}_{\mathrm{EGNOS}}
$$

where:

$\mathrm{dh}$ - difference of $\mathrm{h}$ element value in GPS and EGNOS solutions, $\mathrm{h}_{\mathrm{GPS}}$ - $\mathrm{h}$ coordinate value in GPS solution; $\mathrm{h}_{\text {EGNOS- }} \mathrm{h}$ coordinate value in EGNOS solution. 


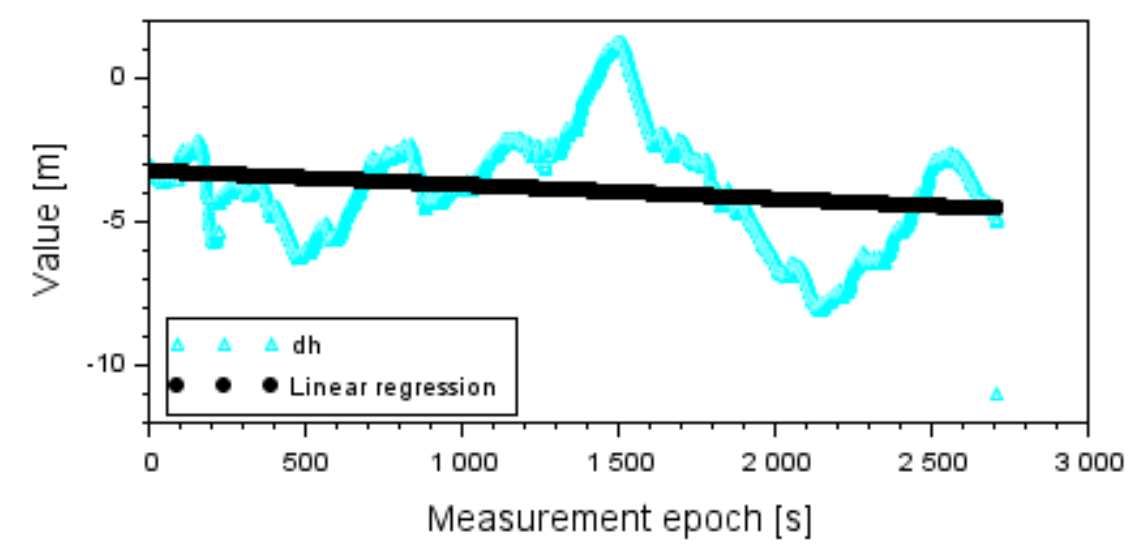

Fig. 4. Difference of ellipsoidal height results based on GPS and EGNOS solutions.

Figure 4 shows the results of comparing the readings for the $\mathrm{h}$ element on the basis of a navigational solution of a position in the tracking mode GPS and EGNOS (SBAS). The average value of the helement difference on the basis of a comparison of solutions GPS and EGNOS is $-3.96 \mathrm{~m}$, with the statistical median parameter being equal to $-3.90 \mathrm{~m}$. Furthermore, the dispersion of the obtained results for the parameter $\mathrm{dh}$ is from $-11.11 \mathrm{~m}$ to $+1.10 \mathrm{~m}$. It should be added that the precision error RMS for parameter dh is equal to $1.94 \mathrm{~m}$. It is worth noting that approximately $27 \%$ of all the parameter values dh is less than $-5 \mathrm{~m}$. It should be added that approximately $67 \%$ of all the dh parameter values is less than $-3 \mathrm{~m}$. In addition, approximately $93 \%$ of all the parameter values $\mathrm{dh}$ is less than $1 \mathrm{~m}$. In turn over $99 \%$ of all the parameter values dh is below $1 \mathrm{~m}$. Figure 4 also specifies a trend of dH parameter changes based on GPS solution and EGNOS (SBAS) solution. For this purpose, the function of linear regression for the characteristics of changes of a first degree polynomial was used. The values of linear coefficients $(a, b)$ of the function of linear regression were designated using the least squares method without the introduction of weighted measurements [10]. Therefore, the linear coefficients $(a, b)$ describing the nature of the changes of dh parameter have the following values: $\mathrm{a}=0.001 \mathrm{~m} / \mathrm{s}$, and $(\mathrm{b})=3.296 \mathrm{~m}$, respectively. In addition, an error matching the function of linear regression with regard to the matching results of the parameter $\mathrm{dh}$ is $\mathrm{m}=1.91 \mathrm{~m}$. The distribution of corrections in the least squares method ranged from $-5.12 \mathrm{~m}$ to $+6.50 \mathrm{~m}$.

On the basis of the conducted investigations, it is possible to verify the usefulness of the test method, presented in this paper, in order to determine the reliability of establishing the position of an aircraft Cessna 172. It should be observed that in the assessment of reliability, the measurement data were registered by only one navigation sensor - the onboard receiver Thales Mobile Mapper. The smallest differences in the readings of coordinate values BLh for the receiver Thales Mobile Mapper can be observed for element L. Therefore, the coordinate values L, in the tracking mode GPS and EGNOS (SBAS), are comparable in the range from - 0.5 to $+3 \mathrm{~m}$. The dispersion of results for the comparison of component B of GPS and EGNOS (SBAS) navigation solutions is larger than for element $\mathrm{L}$. The interval of comparing the readings of coordinate $\mathrm{B}$ in the tracking mode GPS and EGNOS equals $-2 \mathrm{~m}$ to $+6 \mathrm{~m}$. On the other hand, the largest dispersion of results for the comparison of BLh coordinates is visible for the vertical element $h$. The comparison interval of readings of coordinate L in the tracking mode GPS and EGNOS ranges from over $-11 \mathrm{~m}$ to more than $+1 \mathrm{~m}$. It should be added that the biggest differences of the vertical $h$ value occur in the initial measurement epochs during the takeoff phase of the air test. The middle and final phase of the air test, the differences in values of the h element are included in the range from $-8 \mathrm{~m}$ up to approximately $+1 \mathrm{~m}$.

The issue tackled in the research article on the evaluation of the reliability of position determination of the Cessna 172 is a relatively difficult task to tackle and comprises a wide spectrum of knowledge, purely practical one, from the area of implementation of GNSS navigation systems in aviation. The issue of determining a reliable Cessna 172 position is closely related to the elimination or a reduction of major and systematic errors, or specifying random errors during the alignment of GNSS satellite observations. Furthermore, the process of 
algorithmization and the mechanism for determining the position is extremely important in assessing the reliability of the determination of the Cessna 172 coordinates. The research findings, proposed in this paper, concern the use of the device produced by Thales company. It should be noted that in this type of tests, it is possible to use navigation receivers of various classes (A, B or C) or, just the surveying receivers to enable positioning in real time and recording of observations in post-processing [11]. Such an approach of aircraft users will only increase assessment reliability in determining a position of an aircraft. It will also provide a deeper comparative analysis of used sensors in air experiments.

\section{ConClusions}

The aim of the article is to discuss results of reliability assessment of determining the Cessna 172 aircraft in the ellipsoidal BLh system. For this purpose, the navigation data from the onboard receiver Thales Mobile Mapper, recording a position of an aircraft during a test flight over the military aerodrome EPDE in Dęblin, was used. The aircraft position in the lipoidal BLH system was determined based on a navigation solution in the GPS and EGNOS (SBAS) tracking mode. The article discusses the results of comparing the values of designated BLH coordinates of the aircraft Cessna 172 based on the GPS and EGNOS (SBAS) solutions. The average difference in readings the B element between solutions GPS and EGNOS (SBAS) equals $1.17 \mathrm{~m}$, with the RMS error equal to $1.67 \mathrm{~m}$. The average difference in readings the L element between the GPS and EGNOS (SBAS) solutions is $0.75 \mathrm{~m}$, with the RMS error being equal to $0.75 \mathrm{~m}$. The average difference in readings of the $\mathrm{h}$ element between the GPS and EGNOS (SBAS) solutions is $-3.96 \mathrm{~m}$, and the RMS error is equal to the $1.94 \mathrm{~m}$. In addition, the work defines the coefficients of precision dilution PDOP based on the GPS solution and EGNOS (SBAS) solution. The average value of the PDOP coefficient for a solution in the GPS tracking mode equalled 2.7, whereas in the EGNOS (SBAS) tracking mode, it was equal to 2.8.

\section{REFERENCES}

[1] Banaszek K., Malarski M., Required naviation performance and risk of airport operations, Problemy eksploatacji, nr 4, pp. 71-78, 2009. (in Polish)

[2] Kadaj R., How to calculate the GPS measurements?, NAWI, dodatek GEODETY, nr 1 (19), pp. 10-13, 2009. (in Polish)

[3] Wierzbicki D., Krasuski K., Estimation of rotation angles based on GPS data from a UX5 Platform, Measurement Automation Monitoring, Nov. 2015, vol. 61, no. 11, pp. 516-520.

[4] Krasuski K., Application the GPS code observations in BSSD method for recovery the position of the aircraft, Journal of Automation, Mobile Robotics \& Intelligent Systems, vol. 11, no. 3, pp. 45-52, 2017, DOI: 10.14313/JAMRIS_3-2017/27.

[5] Ćwiklak, J., Jafernik, H., The monitoring system for aircraft and vehicles of public order services based on GNSS, Annual of Navigation, no. 16, pp. 15-24, 2010.

[6] Hejmanowska B., Palm Rodolphe, Oszczak S., Ciećko A., Validation of methods for measurement of land parcel areas, Draft final report, AGH University of Science and Technology, pp. 111-113, 2005.

[7] Maciuk K., DOP coefficients in GNSS observations, Budownictwo i Architektura, vol. 14, nr 1, pp. 6572, 2015. (in Polish)

[8] Grunwald G., Ciećko A., Oszczak S., Kaźmierczak R., Grzegorzewski M., Ćwiklak J., The application of EGNOS system in aircrafts monitoring referred to Safety-of-Life service activation, Aparatura Badawcza i Dydaktyczna, nr 3, pp. 133-142, 2011. (in Polish)

[9] Przestrzelski, P., Bakuła, M., Performance of real time network code DGPS services of ASG-EUPOS in north-eastern Poland, Technical Sciences, 17(3), 2014, 191-207. 
[10] Wierzbicki D., Determination of Shift/Bias in Digital Aerial Triangulation of UAV Imagery Sequences, IOP Conf. Series: Earth and Environmental Science vol. 95, World Multidisciplinary Earth Sciences Symposium (WMESS 2017), doi: 10.1088/1755-1315/95/3/032033, pp. 1-9, 2017.

[11] Jafernik H., Krasuski K., Michta J., Assessment of suitability of radionavigation devices used in air, Scientific Journal of Silesian University of Technology. Series Transport, 2016, 90, 99-112. ISSN: 0209-3324. DOI: 10.20858/sjsutst.2016.90.9. (in Polish) 